\title{
Imaging and Pathology Correlations for Different Risk Stratification Models for Intermediate-risk Prostate Cancer
}

\author{
TIMOTHY ELLIS-CALEO ${ }^{1}$, JOHN V. HEGDE ${ }^{1}$, SUKHJEET BATTH ${ }^{2}$, SHANE MESKO ${ }^{3}$, \\ ROBERT REITER $^{4}$, DANIEL MARGOLIS ${ }^{5}$ and MITCHELL KAMRAVA ${ }^{6}$ \\ ${ }^{1}$ Department of Radiation Oncology, University of California, Los Angeles, CA, U.S.A.; \\ ${ }^{2}$ Department of Radiation Oncology, University of Southern California, Los Angeles, CA, U.S.A.; \\ ${ }^{3}$ Department of Radiation Oncology, University of California, Irvine, CA, U.S.A.; \\ ${ }^{4}$ Department of Urology, University of California, Los Angeles, CA, U.S.A.; \\ ${ }^{5}$ Department of Radiology, Weill Cornell Medicine, New York, NY, U.S.A.; \\ ${ }^{6}$ Department of Radiation Oncology, Cedars Sinai Medical Center, Los Angeles, CA, U.S.A.
}

\begin{abstract}
Background/Aim: We evaluated whether substratifying intermediate-risk (IR) prostate cancer using the Memorial Sloan Kettering Cancer (MSKCC) or Prostate Cancer Risk Stratification (ProCaRS) model predicts for adverse imaging or pathologic features. Patients and Methods: 56 consecutive IR patients who underwent multi-parametric MRI (mpMRI) and radical prostatectomy (RP) were studied. The different groups were tested for correlation with adverse findings. 2-sample T-tests assuming unequal variance were used. Results: On mpMRI the MSKCC unfavorable group had higher index lesion suspicion scores $(p=0.044)$, while the ProCaRS model showed a higher maximum tumor diameter (MTD) in the high-risk group $(p=0.047)$. At RP, a higher pathologic MTD (23.3 vs. $17.6 \mathrm{~mm}, p=0.005)$ was present in the MSKCC unfavorable group as well as the ProCaRS high vs. low group (26.6 vs. $19.3 \mathrm{~mm}, p=0.022$ ). Conclusion: Both models demonstrated a correlation with higher MTD for unfavorable IR patients. This is likely a driver of worse clinical outcomes.
\end{abstract}

Prostate cancer is a highly heterogeneous disease which was initially stratified into low, intermediate, and high-risk groups to help guide therapy and determine prognosis (1). Eventually, it became apparent that the three-group risk system needed to be modified given the wide range of

This article is freely accessible online.

Correspondence to: Mitchell Kamrava, MD, Department of Radiation Oncology, Cedars Sinai Medical Center, North Tower, Lower Level, 8700 Beverly Blvd, Los Angeles, CA 90048, U.S.A. Tel: +1 3106593332, Fax: +1 3104231858, e-mail: mitchell.kamrava@cshs.org

Key Words: ProCars, MSKCC, mpMRI, prostatectomy, maximal tumor diameter. clinical outcomes among patients within the same risk group. This is particularly true for men with intermediate-risk prostate cancer, where so-called favorable-intermediate prostate cancer behaves similarly to low-risk disease with regards to prostate cancer-specific mortality (PCSM), while unfavorable-intermediate risk more closely follows high-risk disease $(2,3)$.

The Memorial Sloan Kettering Cancer Center (MSKCC) model (Table I) proposes to divide the intermediate group into "favorable" and "unfavorable" categories (2). Men with a biopsy Gleason score (GS) of $\leq 3+4$ and less than $50 \%$ of biopsy cores positive are classified as having favorable intermediate-risk disease. In contrast, men with a GS $\geq 4+3$ and more than $50 \%$ of biopsy cores positive, or those with multiple risk factors including PSA $10-20 \mathrm{ng} / \mathrm{ml}$, cT2b-c staging, and a GS of 7, are classified as unfavorable intermediate-risk disease.

The prostate cancer risk stratification (ProCaRS) model (Table I) proposes to divide the intermediate-risk group into high- and low-risk categories (4). The low intermediate-risk group contains men with T1-T2 disease and PSA $<10 \mathrm{ng} / \mathrm{ml}$, or men with stage T1-T2 disease, PSA between 10 and 20 $\mathrm{ng} / \mathrm{ml}$, and GS $\leq 6$. The high intermediate-risk group contains men with T2b/c disease or GS 7 with PSA $<20 \mathrm{ng} / \mathrm{ml}$. Table I summarizes the criteria for these two classification systems.

Multiparametric MRI (mpMRI) has shown promise for improving biopsy targeting, staging accuracy, active surveillance program design, image-guidance for treatment delivery, and for predicting adverse pathologic outcomes (510). However, it is unknown whether mpMRI parameters may be associated with the recently established intermediaterisk sub-stratification models which predict PCSM.

In the present study, we investigated whether unfavorable/ high intermediate-risk or favorable/low groups based on the MSKCC and ProCaRS models in men with intermediate-risk 
Table I. A comparison of recent sub-stratification models for intermediate-risk prostate cancer.

\begin{tabular}{ll}
\hline MSKCC Criteria (2) & ProCaRS Criteria (4) \\
\hline $\begin{array}{l}\text { Favorable: } \\
\text { Gleason score (GS) } \leq 3+4 \text { and }\end{array}$ & Low: \\
$<50 \%$ positive biopsy cores & or 2 and PSA $\leq 10 \mathrm{ng} / \mathrm{ml}$ \\
& PSA $\leq 10$ PSA $>10$ and \\
& [T1-T2a or GS $\leq 6]$ \\
& High: \\
Unfavorable: & T1-T2 and PSA $\leq 20 \mathrm{ng} / \mathrm{ml}$ \\
GS 4+3 and $\geq 50 \%$ positive & and \\
biopsy cores or & PSA $>10$ and $[\mathrm{T} 2 \mathrm{~b} / \mathrm{c} \mathrm{OR} \mathrm{GS}>7]$ \\
Multiple risk factors: & \\
1. PSA $10-20 \mathrm{ng} / \mathrm{ml}$ & \\
2. cT2b-c & \\
3. Gleason score 7 & \\
\hline
\end{tabular}

prostate cancer correlate with pre-treatment mpMRI parameters or pathologic specimen information following radical prostatectomy $(\mathrm{RP})$.

\section{Patients and Methods}

Patient selection. This was an Institutional Review Board (IRB)approved retrospective analysis of 56 consecutive men with newlydiagnosed intermediate-risk prostate cancer treated by radical prostatectomy (RP) at the University of California, Los Angeles (UCLA) Medical Center in 2012. All men had intermediate-risk prostate cancer based on National Comprehensive Care Network $(\mathrm{NCCN})$ guidelines, and all underwent 1.5 or 3-Tesla multiparametric MRI (mpMRI) prior to RP as part of surgical planning (11). The mpMRI procedure acquired multiplanar T2 weighted imaging, diffusion weighted and contrast enhanced imaging. Image interpretation was performed on $\mathrm{T} 2$ weighted images. $\mathrm{K}^{\text {trans }}$ values were obtained from images acquired with intravenous gadolinium contrast. Apparent diffusion coefficient maps were created from diffusion weighted images. Additional imaging details have been previously described (12). Based on the MSKCC model, patients were retrospectively stratified into favorable-intermediate and unfavorable-intermediate categories based on pre-treatment clinical and biopsy parameters. They were also retrospectively stratified into low and high intermediate-risk categories based on the ProCaRS model.

Correlation of intermediate-risk prostate cancer stratification models with pre-treatment mpMRI parameters and adverse RP outcomes. Baseline demographic, clinical and biopsy data were recorded for each patient from retrospective medical records review. Additionally, the apparent diffusion coefficient (ADC), capillary permeability ( $\mathrm{k}^{\text {trans }}$ and $\mathrm{k}_{\mathrm{ep}}$ ), iAUC, max tumor diameter (MTD), and MRI suspicion (Likert) score of the largest lesion for each patient were recorded from the clinical mpMRI report. In 2012 our institution was not using the PIRADS system. RP specimen data were also collected, including pathologic T-stage, GS, extracapsular extension (ECE), seminal vesicle invasion, lymphovascular
Table II. Baseline patient demographic, clinical, biopsy, and mpMRI characteristics.

\begin{tabular}{lc}
\hline Mean Age (years) (Range) & $61.9(44.0-77.9)$ \\
Mean PSA (ng/ml) (Range) & $6.4(2.6-56.0)$ \\
Clinical T Stage (Percent) & \\
T1c & $44(78.6 \%)$ \\
T2a & $11(19.6 \%)$ \\
T2c & $1(1.8 \%)$ \\
Biopsy Type (Percent total) & \\
$\quad$ Systematic only & $42(75 \%)$ \\
Targeted only & $0(0 \%)$ \\
Both & $14(25 \%)$ \\
Biopsy Gleason Score (Percent) & \\
3+4 & $39(69.6 \%)$ \\
4+3 & $17(30.4 \%)$ \\
Mean MRI Prostate Volume (cc) (Range) & $36.8(14.0-126.0)$ \\
Mean ADC (10 ${ }^{-6}$ mm $\left.^{2} / \mathrm{s}\right)($ Range) & $1003.7(514-1416)$ \\
Mean K trans (min $^{-1}$ ) (Range) & $0.5(0.04-1.327)$ \\
Mean K ep $_{\text {(min }}^{-1}$ ) (Range) & $1.544(0.141-3.643)$ \\
Mean iAUC (area) (Range) & $10.912(0.246-30.386)$ \\
Mean Maximum Tumor Diameter (mm) (range) & $11.459(5-30)$ \\
\hline
\end{tabular}

$\mathrm{K}^{\text {trans: }}$ Transfer constant, $\mathrm{K}_{\mathrm{ep}}$ : rate constant; PSA: prostate specific antigen; iAUC: area under curve; ADC: apparent diffusion coefficient.

invasion, perineural invasion and mean tumor diameter (MTD). The MSKCC and ProCaRS intermediate-risk stratification models were evaluated for their ability to discriminate between both pretreatment mpMRI parameters and adverse pathologic outcomes following RP.

Statistical analysis. 2-sample T-tests assuming unequal variance and odds ratios (OR) with $95 \%$ confidence intervals (CI) were used to compare sub-group pre-treatment MRI parameters and adverse pathologic outcomes. A $p$-value $<0.05$ was considered statistically significant.

\section{Results}

Baseline clinical, biopsy, and mpMRI characteristics. Table II describes the patient characteristics. The median age of the patients was 62 years (range 44-78). The mean ( \pm standard deviation, SD) PSA was $7.5 \mathrm{ng} / \mathrm{ml}( \pm 3.8) .68 \%$ of patients had a GS of $3+4$, while $32 \%$ had a GS of $4+3$. Clinical stage was $\mathrm{T} 1$ in $79 \%$ of patients and $\mathrm{T} 2$ in $21 \%$. Under the MSKCC classification, $25(44.6 \%)$ of the cohort were classified as favorable intermediate risk with $31(55.4 \%)$ being classified as unfavorable intermediate risk. Under the ProCARS system, $45(80.4 \%)$ were classified as low intermediate risk and 11 $(19.6 \%)$ were classified as high intermediate risk. Of the 31 unfavorable intermediate risk patients in the MSKCC system, 21 were classified as low intermediate risk by ProCARS. A single patient classified as low intermediate risk by ProCARS was classified as unfavorable by MSKCC. The remaining 34 patients were classified as either low and favorable or high 
Table III. Logistic regression analyses evaluating the predictive value of the MSKCC and ProCaRs intermediate risk prostate cancer models to predict for pre-treatment mpMRI parameters or adverse pathologic outcomes.

1. MSKCC stratification

\begin{tabular}{|c|c|c|c|c|}
\hline & Imaging/Pathologic variable & Favorable Inter $(n=25)$ & Unfavorable Inter $(\mathrm{n}=31)$ & $p$-Value \\
\hline \multirow[t]{7}{*}{ mpMRI } & Mean $\mathrm{MTD} \pm \mathrm{SD}(\mathrm{mm})$ & $10.25 \pm 3.40$ & $12.49 \pm 5.61$ & 0.084 \\
\hline & Mean ADC \pm SD $\left(10-6 \mathrm{~mm}^{2} / \mathrm{s}\right)$ & $1048 \pm 197.05$ & $981 \pm 196.81$ & 0.239 \\
\hline & Mean $\mathrm{K}^{\text {trans }} \pm \mathrm{SD}\left(\mathrm{min}^{-1}\right)$ & $0.491 \pm 0.31$ & $0.69 \pm 0.30$ & 0.252 \\
\hline & Mean $\mathrm{K}_{\mathrm{ep}} \pm \mathrm{SD}\left(\mathrm{min}^{-1}\right)$ & $1.57 \pm 0.99$ & $1.51 \pm 0.72$ & 0.832 \\
\hline & Mean iAUC \pm SD (area) & $9.06 \pm 5.51$ & $13 \pm 6.75$ & 0.075 \\
\hline & MRI Suspicion Score & $3.16 \pm 0.96$ & $3.71 \pm 0.94$ & 0.044 \\
\hline & Capsule involved or bulge & $60 \%$ & $68 \%$ & 0.45 \\
\hline \multirow[t]{8}{*}{ RP } & GS upgrade & $8 \%$ & $23 \%$ & 0.13 \\
\hline & Mean MTD \pm SD $(\mathrm{mm})$ & $17.6 \pm 7.44$ & $23.3 \pm 7.31$ & 0.005 \\
\hline & Mean $\% \pm$ SD prostate occupied & $9 \% \pm 4.59$ & $17 \% \pm 12.05$ & $<0.001$ \\
\hline & PNI & $72 \%$ & $90 \%$ & 0.087 \\
\hline & LVI & $0 \%$ & $6 \%$ & 0.35 \\
\hline & SVI & $0 \%$ & $10 \%$ & 0.23 \\
\hline & ECE & $8 \%$ & $39 \%$ & 0.01 \\
\hline & T-stage upgrade from MRI & $40 \%$ & $65 \%$ & 0.07 \\
\hline
\end{tabular}

mpMRI: Multi-parametric MRI, RP: radical prostatectomy, MTD: maximal tumor diameter, ADC: apparent diffusion coefficient, \% prostate: $\%$ occupied by lesion, PNI: perineural invasion, LVI: lymphovascular invasion, SVI: seminal vesicle invasion, ECE: extracapsular extension, iAUC: area under curve, SD: standard deviation.

2. ProCaRs stratification

\begin{tabular}{|c|c|c|c|c|}
\hline & Imaging/Pathologic variable & Low inter $(\mathrm{n}=45)$ & High Inter $(n=11)$ & p-Value \\
\hline \multirow[t]{7}{*}{ mpMRI } & Mean MTD \pm SD $(\mathrm{mm})$ & $10.9 \pm 4.94$ & $13.78 \pm 3.51$ & 0.047 \\
\hline & Mean $\mathrm{ADC} \pm \mathrm{SD}\left(10-6 \mathrm{~mm}^{2} / \mathrm{s}\right)$ & $1032 \pm 202.66$ & $919 \pm 152.82$ & 0.067 \\
\hline & 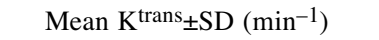 & $0.515 \pm 0.31$ & $0.688 \pm 0.30$ & 0.235 \\
\hline & Mean $\mathrm{K}_{\mathrm{ep}}{ }^{ \pm \mathrm{SD}}\left(\mathrm{min}^{-1}\right)$ & $1.57 \pm 0.95$ & $1.394 \pm 0.12$ & 0.328 \\
\hline & Mean iAUC \pm SD (area) & $9.91 \pm 5.55$ & $15.58 \pm 8.25$ & 0.159 \\
\hline & MRI Suspicion Score & $3.4 \pm 0.99$ & $3.7 \pm 0.95$ & 0.395 \\
\hline & Capsule involved or bulge & $60 \%$ & $73 \%$ & 0.575 \\
\hline \multirow[t]{8}{*}{ RP } & GS upgrade & $18 \%$ & $9 \%$ & 0.49 \\
\hline & Mean MTD \pm SD $(\mathrm{mm})$ & $19.3 \pm 7.07$ & $26.6 \pm 8.36$ & 0.022 \\
\hline & Mean $\% \pm$ SD prostate occupied & $12.23 \pm 8.93$ & $19.1 \pm 14.09$ & 0.147 \\
\hline & PNI & $78 \%$ & $100 \%$ & 0.19 \\
\hline & LVI & $2 \%$ & $9 \%$ & 0.31 \\
\hline & SVI & $4 \%$ & $9 \%$ & 0.54 \\
\hline & ECE & $22 \%$ & $36 \%$ & 0.33 \\
\hline & T-stage upgrade from MRI & $53 \%$ & $55 \%$ & 0.94 \\
\hline
\end{tabular}

mpMRI: Multi-parametric MRI, RP: radical prostatectomy, MTD: maximal tumor diameter, ADC: apparent diffusion coefficient, \% prostate: \% occupied by lesion, PNI: perineural invasion, LVI: lymphovascular invasion, SVI: seminal vesicle invasion, ECE: extracapsular extension, iAUC: area under curve, SD: standard deviation.

and unfavorable intermediate risk by the ProCARS and MSKCC systems, respectively.

Comparison of the intermediate-risk prostate cancer stratification models for pre-treatment mpMRI parameters. Table III describes the results for pre- and post-treatment variables. The MSKCC model significantly predicted for mean \pm SD MRI suspicion score $(3.7 \pm 0.96$ versus $3.1 \pm 0.94$, $p=0.044$ ) for unfavorable versus favorable risk, respectively. The ProCaRS model significantly predicted for mean \pm SD mpMRI maximal tumor diameter $(13.8 \pm 3.5$ vs. $10.9 \pm 5.0 \mathrm{~mm}$, $p=0.047$ ) for high versus low intermediate-risk prostate cancer cohorts, respectively. No other pre-treatment mpMRI parameters, including ADC, Ktrans, Kep, iAUC, or mpMRI evidence of extracapsular bulge/extension, were predicted for by either stratification model. 
Comparison of the intermediate-risk prostate cancer stratification models for adverse pathologic outcomes. Following RP, a statistically significant difference in mean \pm SD maximum tumor diameter (MTD) was seen between the MSKCC unfavorable versus favorable groups $(23.3 \pm 7.3 v s .17 .6 \pm 7.4 \mathrm{~mm}, p<0.005)$. Also, a higher rate of ECE was seen in the MSKCC unfavorable group as compared to the favorable group (39\% vs. $8 \%, p=0.01)$. Furthermore, the percentage of prostate involved with tumor was significantly higher in the unfavorable $v s$. favorable intermediate-risk group $(17 \pm 12.05 \%$ versus $9 \pm 4.59 \%$, $p<0.001)$. The ProCaRS model also had a significantly higher mean $\pm \mathrm{SD}$ maximum tumor diameter in the high $v s$. low intermediate-risk group $(26.6 \pm 8.4 \mathrm{vs} .19 .3 \pm 7.1 \mathrm{~mm}$, $p=0.022$ ).

\section{Discussion}

In the present study of 56 patients with intermediate-risk prostate cancer, both intermediate risk sub-stratification models had variable predictive value for adverse pre-treatment mpMRI features and adverse pathologic specimen outcomes. The MSKCC and ProCaRS intermediate-risk sub-groups both had significantly higher pathologic MTD between favorable/low vs. unfavorable/high intermediate-risk men. The MSKCC stratification showed a significantly higher Likert score for the unfavorable sub-group, while the ProCaRS stratification also showed a larger mpMRI MTD association with the high intermediate-risk sub-group. Additionally, the MSKCC model was associated with both pathologic ECE and percentage of prostate involved with tumor.

The volume of prostate cancer, as measured by evaluating the number (percentage) of biopsy cores positive prior to treatment, the amount of biopsy core positive for malignancy, or the MTD in the pathologic specimen, has been consistently associated with key clinical outcomes in multiple studies. In a cohort of low- and favorable intermediate-risk men treated with definitive radiotherapy, $>50 \%$ biopsy cores positive was significantly associated with worse PCSM (13). In another study of more than 1,000 patients with cT1c-T3N0M0 prostate cancer treated with external beam radiotherapy with or without a high-dose-rate brachytherapy boost, the percentage of cores positive was associated with rates of distant metastasis, cause-specific survival, and overall survival (14). Further reinforcing the prognostic importance of the volume of disease in prostate cancer treated with radiotherapy, the percentage of cancer volume in biopsy cores has been shown to be prognostic for biochemical failure, distant metastasis, cause-specific survival, and overall survival, even in patients treated with dose-escalated external beam radiotherapy (15).

In the post-prostatectomy setting, it has been demonstrated that men with a MTD $>1.4 \mathrm{~cm}$ are at an independently increased risk for biochemical failure, distant metastasis, and prostate cancer-specific survival after salvage radiotherapy (16). In another study, a MTD $\leq 1.2 \mathrm{~cm}$ in the RP specimen showed a low risk of PSA recurrence despite adverse other features being present (17). Therefore, regardless of the treatment modality (external beam radiotherapy with or without high-dose-rate brachytherapy, dose-escalated external radiotherapy, or radical prostatectomy), prostate cancer volume is undoubtedly an important factor to consider for management and prognosis. Therefore, the association of the MSKCC and ProCaRS models to surrogates of prostate volume, including pathologic MTD (both models), percentage of prostate involved with malignancy (MSKCC model), and to pre-treatment mpMRI MTD in the ProCaRS model, may help inform why these models help stratify for PCSM.

Unfortunately, imaging, even with mpMRI, appears to consistently underestimate the size and extent of prostate tumors. For example, a recent radiologic-pathologic correlation study of over 100 men using modern mpMRI demonstrated that pathologic prostate cancer foci had an average diameter $11 \mathrm{~mm}$ longer and a volume three times greater than based on mpMRI prediction (18). This may account for the reason that in our study, pre-treatment mpMRI MTD in the MSKCC model was not significantly different between the sub-groups.

MTD in men with intermediate-risk disease may be relevant to helping choose the appropriate radiation treatment option, with various options including brachytherapy alone, dose-escalated external beam radiotherapy alone, external beam radiotherapy with a brachytherapy boost, or the addition of ADT to external beam radiotherapy or brachytherapy. As noted in the MSKCC study sub-stratifying intermediate-risk men into favorable and unfavorable subgroups, a difference in 8-year PCSM of $0.8 \%$ vs. $4.2 \%$, respectively, was seen in patients treated with dose-escalated external beam radiotherapy (2). However, a recent report in a cohort that underwent brachytherapy demonstrated a smaller difference with 15-year PCSM of $0.6 \%$ for favorable and $2.2 \%$ for unfavorable intermediate-risk men (19).

These data suggest that does-escalated external beam radiotherapy (to approximately 75-80 Gy) may still not achieve a sufficiently high dose for cure. This is especially clear when compared to the benefit that higher doses with brachytherapy alone (or as a boost) can provide for men with unfavorable risk intermediate-risk prostate cancer, who are more likely to have larger tumors as shown by this study. This hypothesis is supported by a study comparing doseescalated radiotherapy with external beam alone to doseescalation with a high-dose-rate brachytherapy boost for men with intermediate-risk disease, which showed improved disease-free survival and overall survival for brachytherapy patients (20). Men with $>50 \%$ cores positive, perineural invasion, or cT2b-c disease had a superior 5-year 
biochemical control of $96 \%$ when treated with a brachytherapy boost compared to $87 \%$ when treated with dose-escalated external beam radiotherapy alone. As ADT also improves outcomes in patients that tend to have larger tumors (unfavorable risk patients), ADT may in fact also be acting as an alternative way to dose escalate - by sensitizing cancer cells to radiation (21).

Some limitations of the present study are apparent. One limitation is its retrospective nature, so selection bias may be present. Another limitation is that this is a study of a limited number of patients, which may have been too small to show the predictive power of these sub-stratification models for several pre-treatment mpMRI parameters or adverse pathologic outcomes. Alternatively, this may suggest that mpMRI may not be as useful a predictor for important clinical outcomes like PCSM, or it may suggest that and the intermediate-risk sub-stratification models have complementary roles in predicting pathologic and clinical outcomes. Additionally, while MSKCC and ProCaRS may be able to stratify patients with intermediate-risk prostate cancer for PCSM, strong predictors of PCSM following RP include adverse pathologic outcomes like GS upgrading, seminal vesicle invasion, and ECE. The variability of these models to predict for these adverse pathologic outcomes may be due to a larger cohort being needed, or it may be due to these models being incomplete and better informed by additional pre-treatment data - perhaps mpMRI MTD. Indeed, it is plausible to even hypothesize that the pre-treatment clinical and biopsy factors sub-stratifying men into favorable/low and unfavorable/high intermediate-risk prostate cancer are really surrogates for prostate tumor volume, and this is why we see the clinical effects of radiation dose escalation (or dose effectiveness increases with ADT radiosensitization) improving clinical outcomes.

In conclusion, we demonstrated that the two recently established prognostic models for sub-stratification of intermediate-risk prostate cancer can successfully predict for pathologic MTD (both schemes). The presence of pathologic extracapsular extension (MSKCC) and percentage of prostate involved in malignancy (MSKCC) MTD is an important prognostic factor for discriminating between favorable and unfavorable intermediate-risk patients, although association with pre-treatment mpMRI MTD was only seen for the ProCaRS model.

The association of unfavorable/high intermediate-risk prostate cancer with a higher MTD may help explain why we observed greater treatment failure when using the same dose for men with unfavorable/high as compared to favorable/low intermediate-risk disease, which appears improved with the use of ADT. Further optimization of pretreatment prostate tumor volume assessment, perhaps with mpMRI or newer imaging modalities, may help improve treatment selection.

\section{Conflicts of Interest}

None.

\section{References}

1 D'Amico AV, Whittington R, Malkowicz SB, Schultz D, Blank K, Broderick GA, Tomaszewski JE, Renshaw AA, Kaplan I, Beard CJ and Wein A: Biochemical outcome after radical prostatectomy, external beam radiation therapy, or interstitial radiation therapy for clinically localized prostate cancer. JAMA 280(11): 969-974, 1998.

2 Zumsteg ZS, Spratt DE, Pei I, Zhang Z, Yamada Y, Kollmeier $M$ and Zelfeskey MJ: A new risk classification system for therapeutic decision making with intermediate-risk prostate cancer patients undergoing dose-escalated external-beam radiation therapy. Euro Urol 64(6): 895-902, 2013.

3 Serrano NA and Anscher MS: Favorable vs unfavorable intermediate-risk prostate cancer: a review of the new classification system and its impact on treatment recommendations. Oncology (Williston Park) 30(3): 229-236, 2016.

4 Rodrigues G, Lukka H, Warde P, Brundage M, Souhami L, Crook J, Cury F, Catton C, Mok G, Martin AG, Vigneault E, Morris J, Warner A, Gonzalez Maldonado S, Pickles T; Genitourinary Radiation Oncologists of Canada (GUROC): The prostate cancer risk stratification (ProCaRS) project: recursive partitioning risk stratification analysis. Radiother Oncol 109(2): 204-210, 2013.

5 Barrett $\mathrm{T}$ and Haider MA: The emerging role of MRI in prostate cancer active surveillance and ongoing challenges. Am $\mathrm{J}$ of Roentgenol 208(1): 131-139, 2017.

6 Moghanaki D, Harris E, Urdaneta AI, Williams M, Kapoor P, Yu J, Schutzer M, Chang $M$ and Hagan M: Multiparametric magnetic resonance imaging guided salvage low-dose-rate brachytherapy for locally recurrent prostate cancer-a 15-year experience. Int J Radiat Oncol Biol Phys 96(2s): E230, 2016.

7 Gibson E, Bauman GS, Romagnoli C, Cool DW, Bastian-Jordan M, Kassam Z, Gaed M, Moussa M, Gómez JA, Pautler SE, Chin JL, Crukley C, Haider MA, Fenster A and Ward AD: Toward prostate cancer contouring guidelines on magnetic resonance imaging: Dominant lesion gross and clinical target volume coverage via accurate histology fusion. Int J Radiat Oncol Biol Phys 96(1): 188-196, 2016.

8 Mertan FV, Greer MD, Shih JH, George AK, Kongnyuy M, Muthigi A, Merino MJ, Wood BJ, Pinto PA, Choyke PL and Turkbey B: Prospective evaluation of the prostate imaging reporting and data system version 2 for prostate cancer detection. J Urol 196(3): 690-696, 2016.

9 Filson CP, Natarajan S, Margolis DJ, Huang J, Lieu P, Dorey FJ, Reiter RE and Marks LS: Prostate cancer detection with magnetic resonance-ultrasound fusion biopsy: The role of systematic and targeted biopsies. Cancer 122(6): 884-892, 2016.

10 Hegde JV, Chen MH, Mulkern RV, Fennessy FM, D'Amico AV and Tempany CM: Preoperative 3-Tesla multiparametric endorectal magnetic resonance imaging findings and the odds of upgrading and upstaging at radical prostatectomy in men with clinically localized prostate cancer. Int J Radiat Oncol Biol Phys 85(2): e101-107, 2013.

11 Mohler JL, Armstrong AJ, Bahnson RR, D’Amico AV, Davis BJ, Eastham JA, Enke CA, Farrington TA, Higano CS, Horwitz EM, Hurwitz M, Kane CJ, Kawachi MH, Kuettel M, Lee RJ, Meeks 
JJ, Penson DF, Plimack ER, Pow-Sang JM, Raben D, Richey S, Roach M 3rd, Rosenfeld S, Schaeffer E, Skolarus TA, Small EJ, Sonpavde G, Srinivas S, Strope SA, Tward J, Shead DA and Freedman-Cass DA: Prostate Cancer, Version 1.2016. J Natl Compr Canc Netw 14(1): 19-30, 2016.

12 Kamrava M, Hegde JV, Abgaryan N, Chang E, Le JD, Wang J, Kupelian PA and Marks LS: Does the addition of targeted prostate biopsies to standard systemic biopsies influence treatment management for radiation oncologists? BJU Int 117(4): 584-591, 2016.

13 D'Amico AV, Renshaw AA, Cote K, Hurwitz M, Beard C, Loffredo $\mathrm{M}$ and Chen $\mathrm{MH}$ : Impact of the percentage of positive prostate cores on prostate cancer-specific mortality for patients with low or favorable intermediate-risk disease. J Clin Oncol 22(18): 3726-3732, 2004.

14 Huang J, Vicini FA, Williams SG, Ye H, McGrath S, Ghilezan M, Krauss D, Martinez AA and Kestin LL: Percentage of positive biopsy cores: a better risk stratification model for prostate cancer?. Int J Radiat Oncol Biol Phys 83(4): 1141-1148, 2012.

15 Vance SM, Stenmark MH, Blas K, Halverson S, Hamstra DA and Feng FY: Percentage of cancer volume in biopsy cores is prognostic for prostate cancer death and overall survival in patients treated with dose-escalated external beam radiotherapy. Int J Radiat Oncol Biol Phys 83(3): 940-946, 2012.

16 Johnson SB, Hamstra DA, Jackson WC, Zhou J, Foster B, Foster C, Song Y, Li D, Palapattu GS, Kunju L, Mehra R, Sandler H and Feng FY: Larger maximum tumor diameter at radical prostatectomy is associated with increased biochemical failure, metastasis, and death from prostate cancer after salvage radiation for prostate cancer. Int J Radiat Oncol Biol Phys 87(2): 275-281, 2013.
17 Rose BS, Chen MH, Zhang D, Hirsch MS, Richie JP, Chang SL, Hegde JV, Loffredo MJ and D'Amico AV: Maximum tumor diameter and the risk of prostate-specific antigen recurrence after radical prostatectomy. Clinical Genitourin Cancer 12(5): e173179, 2014.

18 Priester A, Natarajan S, Khoshnoodi P, Margolis DJ, Raman SS, Reiter RE, Huang J, Grundfest $\mathrm{W}$ and Marks LS: MRI underestimation of prostate cancer geometry: use of patientspecific molds to correlate images with whole-mount pathology. J Urol 197(2): 320-326, 2016.

19 Merrick GS, Butler WM, Galbreath RW, Fiano R and Adamovich E: Stratification of brachytherapy-treated intermediate-risk prostate cancer patients into favorable and unfavorable cohorts: J Contemporary Brachytherapy 7(6): 430-436, 2015.

20 Marina O, Gustafson GS, Kestin LL, Brabbins DS, Chen PY, Ye H, Martinez AA, Ghilezan MI, Wallace M and Krauss DJ: Comparison of dose-escalated, image-guided radiotherapy $v s$. dose-escalated, high-dose-rate brachytherapy boost in a modern cohort of intermediate-risk prostate cancer patients. Brachytherapy 13(1): 5967, 2014.

21 Polkinghorn WR, Parker JS, Lee MX, Kass EM, Spratt DE, Iaquinta PJ, Arora VK, Yen WF, Cai L, Zheng D, Carver BS, Chen Y, Watson PA, Shah NP, Fujisawa S, Goglia AG, Gopalan A, Hieronymus H, Wongvipat J, Scardino PT, Zelefsky MJ, Jasin M, Chaudhuri J, Powell SN and Sawyers CL: Androgen receptor signaling regulates DNA repair in prostate cancers. Cancer Discov 3(11): 1245-1253, 2013.

Received February 7, 2017

Revised February 27, 2017 Accepted February 28, 2017 